\title{
Migración japonesa y guerra: Fernando Hiramuro, un hibakusha mexicano
}

Sergio Hernández Galindo²

Instituto Nacional de Antropología e Historia, México

Tipo de Trabajo: Ensayo

Material original autorizado para su primera publicación en la Revista Académica de la Facultad de Ciencias Sociales de la Universidad de Palermo.

Recibido: 31-3-2015

Aceptado: 7-4-2015

\section{Resumen}

El presente ensayo mostrará trazos de la historia familiar de un emigrante japonés radicado en México. De suyo importante, la vida de Fernando Hiramuro concentrará sin embargo procesos históricos de más largo alcance que llevaron al traslado de cientos de miles de migrantes de ese país al continente Americano. La historia de Toraichi Hiramuro, enmarcada en un periodo de inicios del siglo XX hasta algunos años después del lanzamiento de la bomba atómica sobre la ciudad de Hiroshima en 1945, dará cuenta en principio de la situación que en Japón orilló la expulsión de miles de trabajadores y que al mismo tiempo fueron atraídos por fuerzas económicas que arrastraron a Hiramuro en un principio a Perú, y posteriormente a México. En estos dos países, como en otros de Latinoamérica, se habían insertado flujos enormes de capital extranjero en sectores de su economía como el de la plantación de azúcar y de construcción de vías férreas, pero donde no se contaba con suficiente fuerza de trabajo local. La vida de Hiramuro igualmente mostrará el profundo conflicto que se desató por el control del Pacífico entre Japón y los Estados Unidos y que llevó, por un lado, al lanzamiento de la bomba atómica sobre la ciudad de Hiroshima, donde se encontraban radicando los hijos de Hiramuro que habían nacido en México; y por otro, a la concentración y acoso de las comunidades de japoneses en el continente.

\section{$\underline{\text { Palabras clave }}$}

\footnotetext{
${ }^{1}$ A la memoria del Doctor Fernando Hiramuro.

${ }^{2}$ Magíster en estudios de Asia y África, especialidad en Japón (El Colegio de México). Profesor e Investigador de la Dirección de Estudios Históricos del Instituto Nacional de Antropología e Historia (INAH), México. sergioherg@prodigy. $\underline{\text { net.mx }}$
} 
Japón. México. Migración. Bomba atómica.

\section{Abstract}

The present essay will show lines of the familiar history of a Japanese emigrant taken root in Mexico. Being important, the life of Fernando Hiramuro will concentrate nevertheless historical processes of longer scope that led to the transfer of hundreds of thousands of migrants of this country to the American continent. The history of Toraichi Hiramuro, framed in a period of beginning of the XXth century until some years after the throwing of the atomic bomb to the city of Hiroshima in 1945, will realize at first of the situation that in Japan edged to the expulsion of thousands of workpeople and that at the same time they were attracted by economic forces that dragged Hiramuro in a beginning to Peru, and later to Mexico. In these two countries, as in others of Latin America, enormous flows of foreign capital had been inserted in sectors of its economy as that of the plantation of sugar and of the construction of iron routes, but where one was not provided with sufficient local workforce. The life of Hiramuro equally will show the deep conflict that came untied for the control of the Pacific Ocean between Japan and the United States and that took, on the one hand, to the throwing of the atomic bomb in the city of Hiroshima where there were taking root the children of Hiramuro who had been born in Mexico; and for other, to the concentration and harassment of the Japanese's communities in the continent.

Keywords: Japan. Mexico. Migration. Atomic bomb.

\footnotetext{
망

A principios del año de 2014 murió repentinamente el Doctor Fernando Hiramuro. La muerte se lo llevó de pronto; como de pronto, al estallar la Guerra del Pacífico en 1941, ya no pudo regresar de Japón a la ciudad de Guadalajara, México, donde había nacido. Igualmente, de manera intempestiva en el verano de una mañana calurosa y húmeda en la ciudad de Hiroshima donde residía, el 6 de agosto de 1945 a las 8:15 de la mañana, lo sorprendió la primera bomba atómica lanzada por Estados Unidos contra Japón. Fernando Hiramuro a sus 12 años de edad fue testigo de este acontecimiento: se encontraba formado, junto con sus demás compañeros, en la ceremonia escolar que diariamente se realizaba.
} 
¿Cómo fue que Fernando nació en México? ¿Cómo fue que presenció y sobrevivió a la bomba atómica, convirtiéndose en un hibakusha mexicano? El objetivo de este ensayo es responder a estas preguntas a partir de una visión más amplia de la situación de los migrantes japoneses que llegaron a México por oleadas desde finales del siglo XIX (Ota Mishima, 1985) y que aun cuando vivían en ese país se vieron involucrados en el conflicto entre Estados Unidos y Japón. La Guerra del Pacífico fue como un terrible terremoto que tuvo como epicentro a Japón y Estados Unidos, pero que provocó un extenso tsunami que inundó la vida de cientos de miles de migrantes que habían llegado a América. El caso de la familia Hiramuro da cuenta de uno de esos casos. ${ }^{3}$

\section{Hiramuro y la migración hacia América}

Toraichi Hiramuro, padre de Fernando, nació en el año de 1891 en la prefectura de Hiroshima. Siendo un niño, quedó huérfano al morir su padre cuando apenas cursaba el cuarto grado de la escuela elemental. En esas condiciones, la madre y hermana de Toraichi tuvieron que trabajar para poder sobrevivir, en tanto que el pequeño tuvo que dejar sus estudios y contribuir a la manutención de la familia realizando actividades diversas.

En el año de 1905 Japón derrotó militarmente al imperio zarista, victoria que situó al país Asiático como potencia mundial, y lo hizo parte del tablero de ajedrez de la disputa hegemónica en el que participaban Estados Unidos, Inglaterra y Alemania por el control de la región (Duss, 1983). Empero el triunfo militar que alcanzó Japón se tradujo en un incremento de las cargas impositivas de la población con el propósito de pagar los enormes gastos militares, en mayor desempleo ante la crisis económica posterior a la guerra, y en una mayor miseria. La prefectura de Hiroshima donde vivía Toraichi, empezó a generar en esta primera década del siglo gran cantidad de emigrantes, convirtiéndose en una de las regiones que envió al extranjero a más de 100 mil trabajadores (Kaigaiijutokei, 1994).

Toraichi en ese entonces laboraba en una tienda que vendía utensilios de cerámica propiedad del señor Kikuzo Hirotani. A pesar de que se encontraba a gusto en ese empleo, consideró que tendría un futuro mejor si emigraba a los Estados Unidos. Las historias de éxito que transmitían los diarios y revistas sobre los miles de migrantes que se radicaban en ese país y la mejoría económica que veía de las familias de los emigrantes lo alentaron a tomar tal determinación. Sin embargo; su deseo de

\footnotetext{
${ }^{3}$ Los datos familiares de la familia Hiramuro que se describen en este trabajo me fueron proporcionados por Fernando Hiramuro, su esposa y su hermana en entrevista realizada en la ciudad de Guadalajara en marzo de 2013.
} 
trabajar en Estados Unidos no se pudo realizar debido a que a partir de 1907, mediante el llamado "Acuerdo de Caballeros" entre los gobiernos de EE.UU y Japón, las visas de ingreso a Norteamérica prácticamente se cancelaron y sólo se otorgaban con grandes dificultades para familiares o esposas de los emigrantes ya establecidos, lo que condujo a que los senderos de las poleadas de migrantes se dirigieran al sur del continente. ${ }^{4}$

Esta situación, empero, no detuvo a Hiramuro en su empeño por emigrar. La decisión personal era importante para salir de Japón, pero no era suficiente. Para cumplir su deseo de trabajar en el extranjero se conjuntaron una serie de factores no sólo internos de su país: existían poderosas fuerzas económicas interconectadas a nivel mundial que requerían de gran cantidad de mano de obra y que impulsaban la contratación en particular de trabajadores japoneses no solo en Estados Unidos, sino en diversos países de América Latina. Perú fue el primer país latinoamericano que firmó un Acuerdo de Amistad y Comercio con Japón en el año de 1873, la economía peruana se encontraba en plena expansión y ante la imposibilidad de traer esclavos de África y haber cerrado el ingreso de trabajadores chinos, la política migratoria peruana promovió el ingreso de fuerza de trabajo japonesa. El primer gran contingente de mano de obra de ese país llegó al Puerto del Callao en 1899 integrado por 798 personas, de las cuales 176 eran originarios de Hiroshima. La gran mayoría de trabajadores que arribaron se concentraban en las enormes plantaciones de azúcar y algodón, y eran contratados en su país por compañías japonesas encargadas de reclutar emigrantes y trasladarlos a los países latinoamericanos donde las grandes empresas extranjeras agroexportadoras tenían sus instalaciones. Las compañías reclutadoras japonesas como Morioka, Meiji y Toya fueron las que llevaron a la gran mayoría de trabajadores a las plantaciones peruanas propiedad de la British Sugar Co. ${ }^{5}$

Entre 1899 y 1923, ingresaron a Perú en esas condiciones 18.258 japoneses (Morimoto, 1987), uno de ellos fue Hiramuro quien se incorporó como jornalero en una plantación de azúcar. Las condiciones de trabajo y salariales eran deplorables en estas empresas por lo que Hiramuro se mudó a la ciudad de Lima en el año de 1909 donde realizó trabajos domésticos como el de jardinero. Sin embargo, el objetivo de Hiramuro era trabajar en los Estados Unidos por lo que su estancia en Perú solo se reduciría a ahorrar algún dinero para sustentar sus gastos de traslado a ese país.

\footnotetext{
${ }^{4}$ Para una visión por país de la emigración japonesa hacia América, consultar Kikumura-Yano (2002).

${ }^{5}$ Una indispensable visión de primera mano sobre los trabajadores japoneses en las plantaciones puede verse en Irie (1942).
} 


\section{El traslado de Hiramuro a México}

A fines del año de 1911, contando con dinero suficiente, Toraichi planeó su viaje a Estados Unidos. La empresa naviera japonesa, Toyo Kisen Kaisha, tenía una serie de buques que regularmente atracaban en puertos de Sudamérica y hacían escala en puertos mexicanos. Para trasladarse a Estados Unidos, Hiramuro decidió primero viajar a México, arribando al puerto de Salina Cruz en el mes de enero de 1912. El plan de Hiramuro consistía en cruzar la frontera con Estados Unidos, aun sin visado, como bracero pues sabía que eso era posible a través de las ciudades fronterizas de Tijuana o Mexicali en el norte de México. Con este propósito, abordó nuevamente un barco carguero con destino al puerto de Guaymas, Sonora. En ese punto estaría a solo unos cientos de kilómetros de Estados Unidos e incluso existía una línea troncal de ferrocarril que conectaba a ese puerto con la ciudad fronteriza de Nogales colindante con la ciudad norteamericana del mismo nombre en el estado de Arizona.

Al llegar a ese puerto, Hiramuro se encontró con una situación que cambiaría no sólo sus planes inmediatos, sino que lo marcaría hasta el fin de su vida sin siquiera imaginárselo. En esta población una importante empresa norteamericana, la Southern Pacific ${ }^{6}$, constructora y operadora de diversas líneas de ferrocarril en Estados Unidos y en México, solicitaba personal para el proyecto de construcción de una nueva línea de ferrocarril. Hiramuro decidió solicitar el empleo pues el salario que pagaba la empresa le pareció atractivo, en tanto organizaba su cruce hacia Estados Unidos.

La economía mexicana a finales del siglo XIX y durante la primera década del siglo XX, década en la que estuvo todavía en el poder el general Porfirio Díaz, creció de manera acelerada. En particular, en el estado de Sonora se establecieron importantes enclaves estadounidenses que explotaron los recursos mineros y crearon una red ferroviaria que logró conectar al país con los Estados Unidos. El boom expansivo se concentró principalmente en el sector exportador de la economía por encima de aquel que producía bienes para el mercado interno. Los sectores más dinámicos de la economía estuvieron controlados por el capital extranjero, básicamente británico, aunque de manera acelerada las inversiones estadunidenses crecieron a una velocidad superior al capital inglés. De 1873 a 1910, el total de kilómetros de vías férreas pasó de sólo 572 a 19.205 (Coatsworth, 1976). A decir del presidente Porfirio Díaz "había que enganchar a México a la locomotora del progreso" (Nieves Medina, 2009, p. 20).

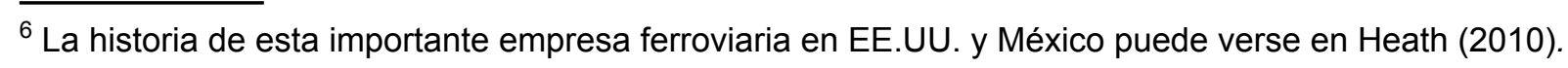


La Southern Pacific, justo cuando llega Hiramuro a México en 1912, requirió para la construcción de la ruta que uniría Guaymas con la ciudad de Guadalajara gran cantidad de mano de obra. La demanda de fuerza de trabajo se había incrementado inesperadamente debido a que escaseaba por el levantamiento armado contra la dictadura del general Díaz que dio inicio a la Revolución Mexicana en 1910. El destierro del presidente Díaz en 1911 no detuvo la revuelta; por contrario, la guerra se generalizaría con el asesinato del presidente Francisco Madero en febrero de 1913. Ante la violencia, las compañías norteamericanas de la región decidieron sacar a su personal extranjero por el conflicto.

La contratación de trabajadores extranjeros en la construcción de los ferrocarriles en esa región no era la excepción sino la norma. En la primera década del siglo XX llegaron al noroeste de México a construir los ferrocarriles migrantes de China y de Japón. El grupo de chinos fue el más numeroso pues sólo en Sonora pasó de poco más de 300 en 1895 a cerca de 5 mil en 1910 (Ota Mishima, 1997). Los japoneses que se concentraron en ese estado llegaron al medio millar, aunque muchos más fueron trabajadores temporales que se movían conforme al avance de los tramos en construcción de las vías férreas por todo el noroccidente de México (Juárez Lucas, 2011).

Hiramuro ingresó como trabajador de esta empresa ferroviaria en el hospital que la Southern había construido para atención de su personal en el poblado de Empalme, Sonora. Hiramuro no sólo tenía gran disposición para trabajar, pues a pesar de no haber terminado sus estudios a nivel elemental, poseía la capacidad y disposición para comprender y desarrollar otras actividades. Su permanencia en la empresa también se debió a que, debido a los enfrentamientos militares, consecuencia de la Revolución en distintos puntos del noroeste, los trabajadores norteamericanos no deseaban trabajar en México por lo que se abrió la posibilidad de que otros trabajadores ocuparan puestos reservados para ellos.

Durante los primeros 15 años de trabajo en la Souhtern, Hiramuro se desempeñó en diversos puestos y actividades: de ayudante de jardinero a jardinero, mensajero, intendente, ayudante de oficina, ayudante de enfermería hasta capacitarse como enfermero en ese hospital. Para 1927 el director del hospital le mencionó que se había inventado una máquina que realizaba unas placas donde se podía observar el interior del cuerpo humano, es decir un aparato de rayos " $x$ ". Para utilizar ese aparato, le propusieron que si quería aprender a manejarlo tendría que tomar un curso de capacitación en Estados Unidos, en un hospital de San Francisco. Sin pensarlo demasiado y sin 
hablar perfectamente el idioma inglés, Hiramuro aceptó de inmediato la propuesta. Los directivos de la Southern Pacific escribieron una carta al cónsul norteamericano solicitándole autorizara un permiso de estancia temporal para Hiramuro.

Al llegar a San Francisco, lo primero que hizo Hiramuro fue trasladarse al barrio japonés para comprar un libro de conversación japonés-inglés y otro de gramática. Con gran esfuerzo y dedicación, estudiando el tiempo que tenía para descansar, avanzó rápidamente en el dominio del idioma, lo que le permitió comunicarse y entender mejor las instrucciones para manejar el aparato de rayos " $x$ ".

En San Francisco su estancia se alargó casi dos años y, a su regreso, la empresa Southern había inaugurado un hospital en la ciudad de Guadalajara mucho mejor equipado que el de Empalme, por lo que Hiramuro fue trasladado a esa ciudad para hacerse cargo del departamento de rayos " $x$ ".

Al inicio de la década de 1930, la situación económica y profesional de Hiramuro era excelente. Atrás habían quedado las etapas de privacidad y de hambre que vivió en Japón. En comparación con la mayoría de migrantes japoneses que residían en México en ese entonces, Hiramuro había logrado construir una carrera laboral exitosa, pues se había especializado como técnico radiólogo con alto reconocimiento y estima en la empresa ferroviaria norteamericana. Por otro lado sus ingresos se habían incrementado sustancialmente de aquel que tenía como empleado de una tienda de cerámica en Japón o como jornalero en la zafra de azúcar en Perú donde apenas ganaba lo esencial para sobrevivir. El salario que disfrutaba era bastante alto no sólo por el nivel de técnico radiólogo, sino que era pagado en dólares por lo que había ahorrado una cantidad considerable de dinero. En esta situación pensó que ya era tiempo de formar una familia pues tenía los medios necesarios para sostenerla de manera holgada en la ciudad de Guadalajara.

Con el propósito de buscar esposa, Hiramuro viajó a Japón al inicio de la década de 1930. Este hecho era realmente extraordinario pues la gran mayoría de los migrantes solicitaban y arreglaban el matrimonio por carta y fotografía desde México sin conocer a su futura pareja, pues no tenían las posibilidades económicas de costear un viaje a Japón, conocido como miaishashin kekkon (Hernández Galindo, 2003). Hiramuro tenía los recursos y las facilidades para realizar este viaje y casarse en Japón, aunque durante su estancia en la ciudad de Hiroshima, donde había nacido, no logró encontrar una muchacha que considerara que podía adaptarse a México y establecer una familia en un país tan diferente del suyo por lo que sin remedio decidió regresar a Guadalajara. 
Unos días antes de su retorno, fue a despedirse del dueño de la tienda de cerámica donde había trabajado, comentándole que no había tenido éxito en conseguir una pareja. El dueño entonces le informó que su hija, Kiyoko Hirotani, era viuda y que aunque tenía una hija pequeña, él y su esposa estarían dispuestos a quedarse con la nieta si él la aceptaba como esposa. Ella era diez años menor y además mucho más preparada escolarmente pues había finalizado sus estudios de preparatoria y realizado un curso para ejercer como maestra de escuela elemental. Hiramuro consideró que una muchacha con esas características estaría preparada para entender mejor el mundo al que ingresaría en México por lo que aceptó la propuesta de su antiguo patrón.

La pareja recién casada regresó a Guadalajara inmediatamente y al año siguiente, en 1931, nació su primer hijo, una niña a quien nombraron Clara Sumie y dos años después nació Fernando Minoru. En 1939, nacería el tercer hijo de la familia, una niña, Concepción Michie. El matrimonio Hiramuro decidió desde el momento en que nacieron sus hijos que sería conveniente que se educaran en Japón, por lo que a los niños en casa siempre se les habló en japonés. Su madre además les enseño a leer y escribir en ese idioma pues no existía una escuela japonesa en Guadalajara como era el caso de las ciudades de México, Navojoa o Mexicali donde se radicaba una comunidad más numerosa de migrantes. Los niños además asistían a la escuela pública por lo que también hablaban el idioma español. El señor Hiramuro con el dinero que había ahorrado y que guardaba en bancos japoneses en la ciudad de Los Ángeles, tenía la capacidad económica para iniciar una nueva vida en Japón, sueño al que aspiraban la mayoría de los migrantes japoneses en México.

\section{Los Hiramuro durante la guerra}

Una primera aproximación para establecerse en Japón de manera definitiva se realizó a fines del año de 1940. La familia completa viajó a Hiroshima con el propósito de que los niños ingresaran a la escuela, aunque Toraichi regresaría a México en 1941 y se reintegraría a su trabajo en el hospital. En Japón Fernando y su hermana Clara habían ingresado a la escuela elemental, sin ninguna dificultad que no se pudiera superar; la pequeña Michie se quedó al cuidado de su madre. La madre los había preparado en México para que se adaptaran a esta nueva situación, además de que los supervisaba y apoyaba cuando algún problema se presentaba, por lo que los niños se integraron adecuadamente al sistema escolar japonés. Al despedirse Hiramuro de la familia y dejarlos en Japón jamás se imaginó que no se verían por muchos años, además de no tener ningún contacto o noticia entre ellos. 
Al estallar la Guerra del Pacífico en diciembre de 1941, la situación de racionamiento para la población se agudizó, ya no solo eran ciertos alimentos y bienes de consumo y materiales, sino en las escuelas escaseaban los lápices y otros materiales. Sin embargo Fernando y Clara, además de resentir la ausencia de su padre, extrañaban la comida y golosinas a las que en Guadalajara estaban acostumbrados. Por otro lado, la situación económica de la familia de su madre, si bien no era boyante, era cómoda a comparación de otros pues a ella le habían dejado una casa que rentaba por lo que tuvo hasta el fin de la guerra un ingreso fijo que le permitió sobrevivir. Las carencias que padecieron no fueron tan graves como aquellas que vivirían después del estallamiento de la bomba atómica y de la ocupación de las fuerzas militares norteamericanas.

Al acercarse la derrota de Japón en 1945, los varones que estudiaban en los niveles de educación básica a partir del tercer grado fueron trasladados en el mes de abril al campo con el propósito de proteger a los niños de los intensos bombardeos que la aviación norteamericana desató sobre las más importantes ciudades de Japón. En el caso de Fernando la escuela se ubicó en un monasterio budista, en el pueblo cercano de Tsuta, como a veinte kilómetros de la ciudad de Hiroshima, donde afortunadamente el templo se encontraba protegido naturalmente por otras montañas. La disciplina y la vigilancia que les impusieron a los estudiantes estuvieron a cargo de un militar quien era el encargado de dar instrucciones en la misma escuela. Hasta ese momento Fernando resintió cierta discriminación de sus compañeros pues sabían que había nacido en el extranjero, hecho que causaba cierta desconfianza en los sectores de la población y en los círculos militares que fomentaban actitudes ultranacionalistas.

En el momento del estallido de la bomba, Fernando se encontraba en la ceremonia escolar, junto con todos sus compañeros, como se acostumbraba cada mañana. Más que el ruido de la explosión atómica, lo primero que sintió fue una intensa luz que lo cegó por unos instantes. Sin saber en realidad que había ocurrido, pues la ciudad no se veía desde ese punto, las horas transcurrieron en esa mañana en una intensa oscuridad como si en realidad hubiera llegado la noche.

Las instrucciones que dieron las autoridades de la escuela después del estallido de la bomba fue que permanecieran en el templo por lo que tuvieron que pasar algunos días para saber de su familia. La única noticia que tuvo de su madre y su hermana fue mediante una carta que ella le envió y donde le informó que se encontraban bien. Pasadas unas semanas después del estallido, su madre pudo visitarlo, y por boca de ella Fernando se enteró de la destrucción completa de la ciudad, hecho 
que era difícil de imaginar. El 15 de agosto a las doce del día se reunió a los estudiantes y a todo el personal para que escucharan un importante mensaje del Tenno Hirohito ${ }^{7}$. El mensaje para los niños y muchos adultos fue incomprensible por las palabras utilizadas en el mismo que no correspondía al lenguaje común y ordinario. La información en ese momento no fue tampoco clara para la mayoría que no entendían que Japón había perdido la guerra. Fernando hasta el siguiente día supo con certeza de la derrota de Japón y que el país se había rendido con la consecuente ocupación militar de las fuerzas norteamericanas, causando una gran decepción en todos los escolares.

En los primeros días del mes de septiembre, Fernando regresó por tren a Hiroshima. Recuerda muy bien que era por la tarde, pues como no había luz eléctrica la ciudad se encontraba en tinieblas. Fue hasta ese entonces que se dio cuenta de la destrucción causada por la bomba atómica, la estación del tren había perdido el techo y el edificio se encontraba carbonizado. De lo que quedaba en pie, solo algunos árboles y postes quemados. Lo que más le llamó la atención a Fernando fue el grupo de niños huérfanos que lo único que hacían era clavarles la mirada para descubrir si identificaban a algún conocido. La escena fue aún más triste pues la lluvia no dejó de caer a lo largo de la tarde y noche de su llegada. Afortunadamente para los Hiramuro, la casa que habitaban no se había destruido o quemado, a pesar de los severos daños que tuvo.

Al día siguiente, sin pasar un momento de descanso, los alumnos se incorporaron a sus clases en lo que nuevamente representó otra sorpresa más para Fernando. La escuela se encontraba destruida en algunas partes. Los primeros días, los alumnos y maestros se dedicaron por completo a limpiar los salones que se encontraban completamente sucios de basura, mezclados con fragmentos de vidrio y madera, además de reparar ventanas y puertas. Hechas estas labores, se reanudaron las clases pero durante algunos meses solo durante las mañanas.

La asistencia matutina a la escuela le permitió a Fernando encargarse de los trabajos de reconstrucción de su propia casa por el resto del día. En ella no sólo habitaban su madre y sus dos hermanas, sino también su abuela. Como jefe de familia, Fernando, auxiliado por unos vecinos y con la ayuda de unos gatos mecánicos, pudo enderezar la casa que quedó ladeada por el impacto atómico. Sin embargo, lo más complicado fue reparar los techos pues existían infinidad de goteras que se filtraban por todas las habitaciones de la casa. Las paredes se pudieron reparar con una malla de bambú que permitía retener los enjarres de tierra que permitieron cubrir las paredes.

\footnotetext{
${ }^{7}$ Es importante usar la palabra en japonés, en vez de emperador, por el carácter sagrado que Hirohito poseía a los ojos de los japoneses.
} 
Fue hasta el año de 1946, gracias a que el correo restableció sus servicios, que el padre de Fernando se enteró que su familia había sobrevivido. Los años que siguieron fueron de duro trabajo para la madre de los Hiramuro, quien se dedicó a coser y elaborar ropa con la máquina Singer que había traído de México, trabajo con el cual pudo mantener a la familia pues ya no tenía ningún ingreso, dado que la casa que tenía en renta quedó destruida por completo. Por otro lado, el señor Hiramuro les empezó a enviar dinero y bultos con comida y ropa cuando las autoridades de ocupación permitieron este tipo de envíos en los siguientes años.

\section{Conclusiones}

El enfrentamiento entre Estados Unidos y Japón no tuvo su escenario exclusivamente en los frentes de batalla militar en el Océano Pacífico que terminaron con el lanzamiento de las bombas atómicas sobre las ciudades de Hiroshima y Nagasaki. Años antes del ataque japonés a la base militar de Pearl Harbor en Hawái en diciembre de 1941, el gobierno norteamericano montó una estrecha vigilancia sobre las actividades de los diplomáticos japoneses y sobre las misiones económicas, culturales y militares que Japón desplegó de manera más activa en los países con los que mantenía relaciones en el Continente.

Pero además, las políticas de vigilancia del gobierno norteamericano en Latinoamérica pusieron énfasis en la observación de las extensas comunidades de emigrantes que se habían establecido de manera mayoritaria en Brasil, Perú, México y Argentina. Los órganos de inteligencia de las embajadas norteamericanas en esos países y el propio FBI, tenían información pormenorizada de los emigrantes, de sus familias, negocios, actividades y en general de su inserción en las sociedades latinoamericanas (Hernández Galindo, 2011). En los primeros meses del año de 1942, en Estados Unidos se ordenó la concentración de los emigrantes japoneses y de sus hijos en campos creados para tal fin; más de 120 mil personas fueron recluidos en esos sitios, incluidos los hijos de los emigrantes que ya eran ciudadanos norteamericanos.

En México, a petición del gobierno norteamericano, los emigrantes y sus familias, aunque no fueron recluidos en campos de concentración, se les ordenó ser concentrados en las ciudades de México y Guadalajara, ubicadas en el centro del país, lejanas de la frontera con Estados Unidos. Las comunidades de japoneses en México, de este modo, afrontaron un tipo de bomba atómica que destruyó de pronto sus hogares, sus medios de vida y los lazos sociales que habían construído en 
los poblaciones donde tenían décadas de haberse establecido.

Toraichi Hiramuro, para su suerte, vivía en la ciudad de Guadalajara, lo que le permitió afrontar la guerra que se desató contra la emigración sin dejar su trabajo y su casa. El regreso de la familia Hiramuro a México no fue fácil, pues aunque los hijos poseían la nacionalidad mexicana y el señor Hiramuro ya se había naturalizado mexicano, las relaciones entre ambos países permanecían rotas. Fue mediante la embajada de Suecia, que era la encargada de los asuntos de México con Japón, la que les expidió el pasaporte en el año de 1950 para que pudieran salir del país mediante la autorización del Supremo Comando que aún seguía ocupando Japón. En un buque de bandera norteamericana la familia zarpó de Yokohama con destino a San Francisco. En ese puerto Toraichi los esperaría después de diez años de separación. La reintegración de la familia Hiramuro a México no fue un proceso sencillo, el regreso al lugar en el cual habían nacido los pequeños representó nuevos retos que afrontar. 


\section{Archivos}

Archivo General de la Nación, México. Fondo Registro Nacional de Extranjeros.

\section{Referencias Bibliográficas}

Coatsworth, J. H. (1976). El impacto económico de los ferrocarriles en el Porfiriato. Crecimiento y Desarrollo. México: SepSetentas.

Duss, Peter (1983). The Takeoff Point of Japanese Imperialism. En Harry Wray y Hilary Conroy (editors) Japan Examined: Perspectives on modern Japanese history (pp. 153-157). Honolulu: University of Hawaii Press.

Heath, E. (2010). Seventy-Five Years of Progress. An Historical Sketch of The Southern Pacific 1869-1944. http://cprr.org/Museum/SP 1869-1944/index.html

Hernández Galindo, S. (2003). Las empresas de los inmigrantes japoneses en México. El caso de la familia Kasuga. En Rosa María Meyer y Delia Salazar (coordinadoras) Los inmigrantes en el mundo de los negocios. México: Plaza y Valdes.

-(2011), La guerra contra los japoneses en México durante la Segunda Guerra Mundial. Kiso Tsuru y Masao Imuro, migrantes vigilados. México: Itaca.

Irie, T. (1942). Hojin Kaigai Hattenshi (Historia del desarrollo al exterior de la migración japonesa). Tokio: lida Shoten, 2 vols., cap. XIV y XV vol. 1.

Juárez Lucas, P. (2011). Trabajadores extranjeros en los ferrocarriles del noroccidente de México 1901-1912. Mirada Ferroviaria, 13, 31-34.

Kaiga liju tokei. 1994. Estadísticas de la migración al extranjero. Año 27 de Meiji-año 5 de Heisei. Tokio.

Kikumura-Yano, A. (2002). Encyclopedia of Japanese descendants in The Americas. New York: Altamira Press.

Morimoto, A. (1987). Población de origen japonés en el Perú. Investigación y Bibliografía. Compilador. Primer Seminario sobre poblaciones inmigrante. Lima: Consejo Nacional de Ciencia y Tecnología.

Nieves Medina, A. (2009). Los ferrocarriles en Sonora. Mirada Ferroviaria, 7, 20-29.

Ota Mishima, M. E. (1985). Siete Migraciones Japonesas en México 1890-1978. México: El Colegio de México.

(1997). Destino México. Un estudio de las migraciones asiáticas a México, siglos XIX y XX. México: El Colegio de México. 